\title{
Atitudes acerca do Aconselhamento Online e o Aconselhamento Face a Face
}

\section{Attitudes about Online Counselling and Face-to-Face Counselling}

\author{
José Tomás da Silva *, Teresa Sousa Machado*, José Pacheco Miguel* \\ *Universidade de Coimbra
}

\begin{abstract}
Resumo
O aconselhamento via Internet é uma prática profissional com uma curta história mas com comprovadas potencialidades de aplicação nas áreas do aconselhamento e da psicoterapia. Um domínio ainda inexplorado diz respeito às atitudes dos clientes acerca desta nova modalidade de aconselhamento. O presente estudo mostra que é possível medir válida e fiavelmente duas facetas atitudinais: valor e desconforto. A amostra deste estudo é constituída por 242 respondentes adultos de ambos os sexos. A validade estrutural bidimensional do instrumento foi comprovada através de uma análise de componentes principais dos itens e a consistência interna dos resultados é muito boa. As implicações práticas dos resultados são discutidas. Palavras-chave: aconselhamento online, aconselhamento face a face, Internet, atitudes, validade
\end{abstract}

\begin{abstract}
Counselling via Internet is a professional practice with a short history but with proven application potential in the areas of counselling and psychotherapy. An unexplored topic concerns client attitudes toward this type of intervention compared to more traditional forms of counselling and psychotherapy. The sample comprises 242 adult respondents of both sexes. The hypothesized structural validity of the instrument was confirmed by a principal components analysis of the ten items and the internal consistency of the results is very good. The practical implications of the results are discussed.
\end{abstract}

Keywords: online counselling, face to face counseling, Internet, attitudes, validity

O aconselhamento via Internet (ou online) é uma prática profissional com uma curta história mas com comprovadas potencialidades de aplicação nas áreas do aconselhamento e da psicoterapia. Como refere Barak (1999) “o aparecimento da tecnologia da informação [nos anos 1980], o seu uso muito difundido a nível mundial devido ao custo razoável que lhe está associado, e o sucesso relativo dos "computadores amigáveis para o utilizador", tornando possível o uso dos computadores por mais pessoas, estão significativamente refletidos na psicologia [atual] ” (p. 231). Se os avanços tecnológicos de início apenas afetaram operações e procedimentos a nível individual e local isso mudou muito rapidamente quando atividades de grupos e de comunidades mais vastas foram incluídas ao serem introduzidas as redes globais de computadores. Como sublinha Barak (1999, p. 231), "a constituição da Internet - uma rede global de computadores que interliga um crescente número de redes e computadores locais - realmente criou uma 'aldeia global', onde as pessoas comunicam e interagem online com facilidade". A tecnologia, e, em especial, as tecnologias da informação e da comunicação (TIC), afetam crescentemente o modo como os psicólogos e, particularmente, os profissionais da saúde mental conduzem o seu dia-a-dia laboral (Anthony, 2003). Em termos históricos as primeiras tentativas sérias para usufruir a Internet com fins terapêuticos foram realizadas em meados dos anos 1990. Nessa altura, o email era a principal forma de tecnologia usada para o estabelecimento da comunicação entre os profissionais e os seus clientes. Hoje, os psicólogos desenvolvem novas aplicações e métodos criativos para disponibilizarem os seus serviços. Como se afirma, numa revisão recente sobre intervenções psicológicas baseadas na Internet, da Australian Psychological Society (APS, 2012), "há um enorme potencial para ampliar o acesso do público a serviços de saúde e bem-estar baseados em evidência científica, assim como oferecer aos psicólogos maior discricionariedade e flexibilidade na disponibilização de serviços a populações às quais anteriormente teriam dificuldade em chegar" (p. 4). Outras vantagens têm sido apontadas como decorrentes da incorporação da tecnologia nas intervenções psicológicas. Por exemplo, a comunicação profissional entre duas partes pode ser mais rápida, mais eficiente e mais conveniente do ponto de vista administrativo; o uso de telemóveis permite o acesso à rede na maioria das zonas mundiais, mesmo as mais remotas. Os novos modos e meios de comunicação, sobretudo, permitem aos clientes disfrutar de tratamentos rentáveis em termos de custos-benefícios quando, de onde, e da maneira que querem. Os psicólogos que pratiquem online podem usufruir de benefícios similares aos dos seus clientes, decidindo quando, onde, e como querem prestar um serviço a um cliente (APS, 2012, p. 4). A internet inclui uma vasta gama de meios e serviços. Barak, Klein e Proudfoot (2009), em particular, distinguem quatro tipos de intervenções baseadas na Internet: intervenções baseadas na web (v.g., intervenções educativas na web, intervenções terapêuticas autodirigidas na web e intervenções terapêuticas na web com apoio humano), "aconselhamento online" (v.g., através de email, ou formas protegidas de chat baseado, ou não, em vídeo), software terapêutico a funcionar independentemente na 
Internet (v.g., simulação robótica, jogos e ambientes virtuais) e, outras atividades online, incluindo blogs, podcasts, grupos de apoio e processos de autoavaliação.

As intervenções psicológicas na Internet apoiam-se em distintas tecnologias (v.g., computador, telemóvel, webcam) e podem ser tanto síncronas como assíncronas.

O avanço tecnológico e o uso generalizado da Internet possibilitaram um acesso mais disseminado dos clientes ao aconselhamento online e a outras formas de intervenções psicológicas na Internet. A enorme generalização no acesso à Internet, verificada nos últimos anos, permite atenuar, embora não resolvendo definitivamente, a questão do "digital divide”, ou dos ditos info-excluídos, uma questão que expõe um óbice recorrentemente esgrimido por quem aponta limitações às intervenções na Internet. O século vinte e um está rapidamente a tornar-se uma era de consumidores esclarecidos que utilizam a tecnologia disponível mais avançada para assumirem o controlo sobre a sua própria saúde, finanças, carreira profissional, comunicações e outras dimensões da sua vida pública e privada. As estatísticas internacionalmente disponíveis sobre o uso dos computadores e, em particular, da Internet, comprovam essa tendência. Em Portugal, em 2014, a percentagem média dos agregados familiares com acesso à internet, através de banda larga é 63\%. Todavia, 98\% dos indivíduos do grupo etário dos 16-24 anos afirmavam utilizar a Internet. Essa percentagem, porém, decai fortemente com a idade: 94\% para grupo etário dos 25-34 anos, 84\% no grupo dos 35-44 anos, 60\% no grupo dos 45-54 anos, 39\% no grupo dos 55-64 anos e apenas 23\% para o grupo etário dos 65-74 anos (Fonte: INE/PORDATA).

Várias investigações empíricas realizadas nas duas últimas décadas e, em particular, os estudos de meta-análise atestam a eficácia das intervenções baseadas na web e, em particular, do aconselhamento via Internet (v.g., Andersson \& Cuijpers, 2009; Barak, Hen, Boriel-Nissim, \& Shapira, 2008; Rochlen, Zack, \& Speyer, 2004; Tate, Finkelstein, Khavjou, \& Gustafson, 2009). Em Portugal, porém, os estudos sobre intervenções psicológicas via Internet são ainda escassos (v.g., Taveira \& Silva, 2011). Como notam Goss \& Anthony (2003) “a ideia da tecnologia intervir de alguma maneira no processo de aconselhamento e psicoterapia parece [ainda] provocar fortes reações [tanto nos profissionais, como nos clientes] ” (p. 1). A disparidade de reações podem tomar um sentido nesta "era digital” se pensarmos que neste momento temos, por um lado, toda uma geração que desde sempre viveu com a internet, telemóveis e computadores a par com gerações de pessoas mais idosas que apenas numa fase mais tardia das suas vidas tomaram contato com as novas tecnologias. Ademais é importante reconhecer que esse contato, para muitos destes adultos, nem sempre foi uma experiência positiva (por exemplo, a automatização proporcionada pela emergência do computador e da telemática conduzindo a alterações significativas nos processos tradicionais de trabalho, ou, ainda a extinção de postos de trabalho). Ora, pode-se especular que a idade pode ser uma variável relacionada com o interesse que os indivíduos revelam em procurar ajuda psicológica através de intervenções baseadas na Internet. Esta questão é particularmente relevante, neste momento, em Portugal uma vez que muitas pessoas precisam de apoio psicológico na área da saúde mental devido às condições adversas de vida decorrentes da profunda crise económica em que nos encontramos. O uso da Internet para proporcionar serviços de aconselhamento psicológico online ao vasto número de adultos desempregados atualmente existentes seria um meio eficaz para alargar os benefícios da ajuda psicológica a indivíduos que dela necessitam, mas que os serviços tradicionais, são incapazes de satisfazer. Todavia, tendo em conta o que dissemos acima, sabemos que nem todos os indivíduos poderão estar igualmente interessados em recorrer a intervenções na Internet, donde é importante saber quais de entre eles têm atitudes positivas versus negativas a respeito desta modalidade de intervenção. A investigação das atitudes dos clientes para com estas novas modalidades de intervenção comparativamente a formas mais tradicionais de aconselhamento e de psicoterapia é um domínio ainda insuficientemente explorado (v.g., Rochlen, Beretvas, \& Zack, 2004).

Uma atitude consiste na avaliação de um objeto atitudinal (pessoa, lugar, coisa, acontecimento), podendo ir de um extremo muito negativo a um extremo muito positivo. As atitudes são importantes determinantes do comportamento. Atitudes fortes a respeito de um objeto são especialmente interessantes, pois, como se sabe, tendem a ser estáveis no tempo, resistentes a tentativas de persuasão e constituem bons preditores do comportamento futuro (Ajzen, 2001). A ausência de escalas que operacionalizem este construto contribui para a assinalada falta de investigações nesta temática. $\mathrm{O}$ presente estudo procura colmatar esta falha mostrando que é possível medir válida e fiavelmente duas facetas das atitudes dos clientes acerca do aconselhamento online e do aconselhamento tradicional: valor e desconforto (Rochlen et al., 2004). Um segundo objetivo, deste estudo exploratório, consiste em determinar hipotéticas diferenças género nas atitudes dos indivíduos a respeito da modalidade de aconselhamento online versus aconselhamento face-a-face. Um terceiro, e último objetivo, visa examinar as relações das atitudes acerca do aconselhamento (online e face-a-face) com a idade.

\section{Método}

\section{Participantes}

A amostra deste estudo é constituída por 242 respondentes de ambos os sexos (cento e nove do sexo feminino e 133 do sexo masculino). A média das idades é de 37.2 anos $(D P=13.2$ anos; mínimo = 18 anos; máximo $=65$ anos). A maioria dos respondentes tem o $9^{\circ}$ ano de escolaridade (55\%), 31\% têm o ensino secundário, 7\% dos restantes respondentes obtiveram o $1^{\circ}$ ciclo, $4 \%$ o $2^{\circ}$ ciclo e apenas $3 \%$ afirmaram possuir algum tipo de formação terciária. 


\section{Instrumentos}

Um breve questionário sociodemográfico foi construído para este estudo incluindo questões sobre o sexo, idade, e as habilitações literárias dos respondentes.

Duas curtas escalas de atitudes (aconselhamento online [Online Counseling Attitudes Scale: OCAS] e face-a-face [Face-to-Face Counseling Attitudes Scale: FCAS]), com dez itens cada, foram traduzidas do Inglês para o português Europeu. Ambas as escalas foram desenvolvidas originalmente por Rochlen et al. (2004). Inicialmente cada um dos autores contribuiu com 30 itens concebidos para avaliar uma variedade de potenciais atitudes acerca dos serviços de aconselhamento online. Para cada um dos itens destinado a avaliar as atitudes a respeito do aconselhamento tradicional foi derivado um item equivalente para o aconselhamento online. Para a forma experimental, depois de terem sido removidos vários itens redundantes ou ambíguos, foram retidos 52 itens (26 para a OCAS e 26 para a FCAS). Estes itens foram administrados num estudo piloto a uma amostra de 471 estudantes universitários, subsequentemente divida em duas subamostras aleatórias com cerca de metade dos respondentes, e as respostas aos itens foram examinadas através de uma análise exploratória de componentes principais (CP) e, subsequentemente, através de análises fatoriais confirmatórias. Em ambas as amostras foram extraídos dois componentes contendo apenas os 10 itens com cargas fatoriais superiores a .5. Os fatores da escala OCAS, na primeira subamostra, explicaram 64.6\% da variância total (na segunda amostra, a percentagem total explicada foi de $62.2 \%$ ). No total foram retidos cinco itens para medir os dois fatores de cada subescala. Os fatores foram denominados Desconforto com o aconselhamento online (OC-D) e Valor do aconselhamento online (OC-V). A subescala OC-D representa os sentimentos e as reações dos participantes quanto ao grau de à vontade e conforto associado com o uso de serviços de aconselhamento online (v.g., "Eu teria pavor em explicitar os meus problemas pessoais a um conselheiro na Internet”). A OC-V é representativa dos sentimentos gerais dos participantes a respeito da utilidade do aconselhamento online (v.g., "A utilização do aconselhamento à distância (Internet) ajudar-me-ia a conhecer-me melhor"). Os mesmos10 itens foram usados para medir as atitudes dos participantes a respeito do aconselhamento face-a-face. Duas subescalas foram igualmente construídas e denominadas, respetivamente, Desconforto com o aconselhamento face-a-face (FC-D) e Valor do aconselhamento face-a-face (FC-V). As respostas foram dadas numa escala de tipo Likert com seis pontos $(1=$ discordo fortemente; $6=$ concordo fortemente). O exame subsequente, da estrutura fatorial de ambas subescalas através da análise fatorial confirmatória (AFC) [vide Rochlen et al., 2004, estudo 2] revalidou os resultados obtidos através das análises fatoriais exploratórias. A consistência interna das respostas (coeficiente alfa) nas duas subescalas é, em geral, boa (os valores oscilaram entre .69 [FC-D: estudo $3, n=213$ ] e .90 [FC-V: estudo $1, n=461])$. Outros dados, designadamente sobre a precisão teste-reteste e estimativas da validade de construto da OCAS e da FOCAS são apresentados por Rochlen et al. (2004).

\section{Procedimento}

Os participantes foram recrutados em quatro delegações do Instituto de Emprego e de Formação Profissional (IEFP) da região Centro e da zona de Lisboa e do Vale do Tejo. Todos os respondentes se encontravam desempregados na altura em que responderam ao questionário. A participação no estudo foi estritamente voluntária tendo-se garantida a confidencialidade das respostas.

\section{Resultados}

As matrizes de intercorrelações (coeficientes de Pearson) para os dois subconjuntos de 10 itens (itens das escalas OCAS e FCAS, respetivamente) foram inicialmente examinadas com o objetivo de determinar a sua adequação para o procedimento de análise exploratória de componentes principais (AECP).

De seguida os 10 itens da OCAS foram sujeitos a uma análise de componentes principais (ACP) usando o programa IBM SPSS Statistics, versão 22.

A análise de componentes principais revelou a presença de dois componentes com valores próprios superiores a um, explicando $31.7 \%$ e $26.7 \%$ da variância, respetivamente. O exame do screeplot revelou uma quebra após o segundo componente. O teste scree de Catell mostrou-se consistente com a regra de Kaiser (valores próprios, ou eigenvalues, maiores que um) para determinação do número de componentes. Ambas análises foram corroboradas pelos resultados de uma Análise Paralela a qual revelou apenas dois componentes com valores próprios excedendo os valores de critério correspondentes gerados para uma matriz aleatória de dados com a mesma dimensão (10 variáveis $\times 242$ respondentes). Para auxiliar a interpretação dos dois componentes foi executada uma rotação Promax. A solução obtida (vide Tabela 1) após rotação mostrou a presença de uma estrutura simples, com ambos componentes incluindo cargas fatoriais (loadings) fortes e todos os itens saturando fortemente em apenas um componente.

Tabela 1.

Coeficientes Padrão/Estrutura para os itens da OCAS

\begin{tabular}{|c|c|c|c|c|}
\hline \multirow[t]{2}{*}{ Item } & \multicolumn{2}{|c|}{ Padrão } & \multicolumn{2}{|c|}{ Estrutura } \\
\hline & CP1 & CP2 & CP1 & CP2 \\
\hline OCAS 1 & -.132 & .656 & -.190 & .668 \\
\hline OCAS 2 & .634 & .055 & .629 & -.001 \\
\hline OCAS 3 & .014 & .714 & -.050 & .712 \\
\hline OCAS 4 & .737 & .103 & .728 & .038 \\
\hline OCAS 5 & .043 & .804 & -.028 & .801 \\
\hline OCAS 6 & .743 & -.079 & .750 & -.145 \\
\hline OCAS 7 & .075 & .803 & .004 & .797 \\
\hline OCAS 8 & .858 & -.062 & .864 & -.138 \\
\hline OCAS 9 & -.002 & .790 & -.072 & .790 \\
\hline OCAS 10 & .840 & -.009 & .841 & -.083 \\
\hline
\end{tabular}


A interpretação dos dois componentes é consistente com a estrutura proposta pelos autores do instrumento. Os itens pares avaliam a atitude de desconforto com o aconselhamento online (todos saturando fortemente no CP1) e os itens ímpares avaliam a atitude respeitante ao valor atribuído ao aconselhamento online (CP2).

Resultados muito idênticos aos relatados acima foram obtidos para os itens que compõem a FCAS (vide Tabela 2).

Os diversos métodos usados para determinar o número de componentes (regra de Kaiser, teste Scree de Catell e Análise Paralela) foram unânimes em apontar uma solução fatorial simples com dois componentes principais explicando, neste caso, 38.9\% e $22.1 \%$ da variância, respetivamente. Uma diferença entre as duas soluções fatoriais é que no caso da FCAS os itens ímpares (indicadores do valor atribuído ao aconselhamento face-a-face) saturaram no primeiro Componente Principal, enquanto os itens relativos ao grau de desconforto saturaram no segundo componente.

Tabela 2.

Coeficientes Padrão/Estrutura para os itens da FCAS

\begin{tabular}{lrrrr}
\hline Item & \multicolumn{2}{c}{ Padrão } & \multicolumn{2}{c}{ Estrutura } \\
\cline { 2 - 5 } & CP1 & CP2 & CP1 & CP2 \\
\hline FCAS 1 & .727 & -.068 & .744 & -.258 \\
FCAS 2 & -.037 & .529 & -.175 & .538 \\
FCAS 3 &. $\mathbf{8 4 3}$ & .048 &. $\mathbf{8 3 1}$ & -.173 \\
FCAS 4 & -.011 & .757 & -.209 &. $\mathbf{7 6 0}$ \\
FCAS 5 & $\mathbf{. 8 1 6}$ & -.013 & $\mathbf{. 8 1 9}$ & -.227 \\
FCAS 6 & -.080 & .746 & -.276 &. $\mathbf{7 6 7}$ \\
FCAS 7 & $\mathbf{. 8 3 6}$ & -.009 & $\mathbf{. 8 3 8}$ & -.228 \\
FCAS 8 & .026 & $\mathbf{. 8 4 3}$ & -.195 & $\mathbf{. 8 3 6}$ \\
FCAS 9 & $\mathbf{. 8 6}$ & .017 & $\mathbf{. 7 8 1}$ & -.189 \\
FCAS 10 & .071 & $\mathbf{. 8 5 3}$ & -.152 & $\mathbf{. 8 3 4}$ \\
\hline
\end{tabular}

De seguida procedemos ao cálculo da consistência interna (coeficiente $\alpha$ de Cronbach) para os construtos Desconforto e Valor (aconselhamento online e face-a-face). Todas as estimativas de consistência interna se revelaram muito boas, com os coeficientes localizando-se nos .80's: .86 (FCAS-V), .81 (OCAS-V), .82 (OCAS-D) e .80 (FCAS-D).

As comparações das médias nas subescalas tomando em consideração o sexo do respondente (vide Tabela 3) foram efetuadas através de testes $t$ Student, não tendo sido encontradas diferenças estatisticamente significativas em qualquer um dos contrastes realizados ( $p$ ’> .05, testes bilaterais).

Tendo em conta que os indivíduos responderam a todas as subescalas torna-se viável examinar o perfil diferencial das suas atitudes relativamente às duas modalidades de aconselhamento (online versus face-a-face). Como anteriormente não encontrámos evidências de diferenças sexuais, as respostas foram analisadas para a totalidade da amostra. Para facilitar a interpretação dos resultados as médias para as subescalas foram previamente estandardizadas (dividindo o score total pelo número de itens correspondentes).
Tabela 3.

Médias e desvios-padrão nas subescalas OCAS e FCAS em função do sexo

\begin{tabular}{lccccc}
\hline \multirow{2}{*}{ Subescala } & \multicolumn{2}{c}{ Masculino $(n=133)$} & & \multicolumn{2}{c}{ Feminino $(n=109)$} \\
\cline { 2 - 3 } \cline { 5 - 6 } & $M$ & $D P$ & & $M$ & $D P$ \\
\hline OCAS-V & 12.83 & 5.31 & & 12.90 & 5.70 \\
OCAS-D & 17.63 & 6.27 & & 16.16 & 7.22 \\
FCAS-V & 19.24 & 6.62 & & 20.80 & 5.59 \\
FCAS-D & 16.03 & 6.34 & & 15.90 & 5.67 \\
\hline
\end{tabular}

Como mostra a Figura 1, os respondentes atribuem mais valor ao aconselhamento face-a-face $(M=3.99 ; D P$ $=1.24)$ do que ao aconselhamento online $(M=2.57$; $D P$ $=1.08$ ). No que diz respeito ao grau de desconforto os respondentes apresentam na subescala de aconselhamento online uma média mais elevada ( $M=$ 3.39; $D P=1.35$ ) do que aquela que referem para o aconselhamento face-a-face $(M=3.19 ; D P=1.21)$. Todavia, apenas o primeiro contraste mencionado é estatisticamente significativo $(t(241)=-16.05, p<.001)$.

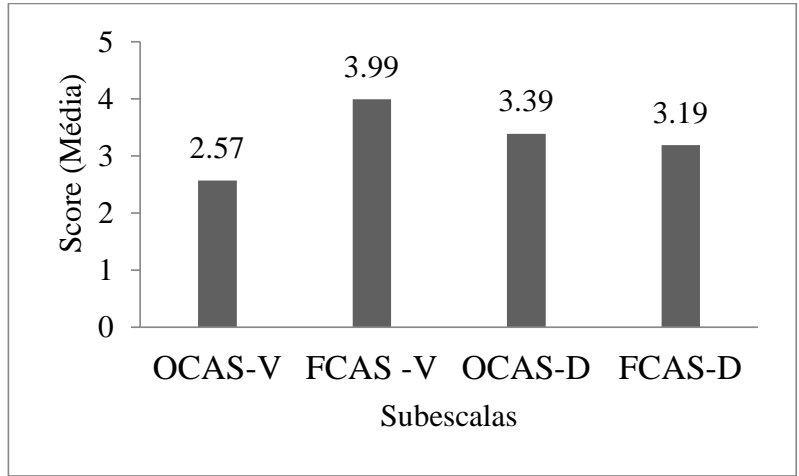

Figura 1. Scores (médias) nas subescalas atitudinais (Valor e Desconforto do aconselhamento online vs. face-a-face)

Finalmente procedeu-se à análise da relação da idade com os scores nas subescalas de atitudes a respeito do aconselhamento.

Encontrámos uma relação positiva pequena, mas estatisticamente significativa, entre a idade e o valor atribuído pelos respondentes ao aconselhamento face-a-face, $r(242)=.15, p=.023, r^{2}=.025$. Já o valor atribuído ao aconselhamento online não mostrou correlacionar com a idade, $r(242)=-.02, p=.82, r^{2}=$ .0004 . No que respeita ao grau de desconforto com o aconselhamento online verificámos que há uma correlação negativa pequena com a idade, $r(242)=-.13$, $p=.048, r^{2}=.017$, enquanto o desconforto com aconselhamento face-a-face parece ser independente da idade, $r(242)=.01, p=.84, r^{2}=.0001$.

\section{Discussão}

As atitudes são importantes determinantes do comportamento, i.e., do que as pessoas pensam, sentem e fazem em múltiplas esferas das suas vidas (Ajzen, 2001). As intervenções psicológicas via Internet (Barak, 1999) são serviços cada vez mais disponibilizadas pelos 
psicólogos aos seus clientes. Apesar disso a possibilidade de efetuar aconselhamento online tem constituído um dos tópicos mais calorosamente discutidos entre os profissionais da saúde mental nas últimas três décadas (Rochlen et al., 2004). Em Portugal, contrariamente ao que se passa nos países anglo-saxónicos, a investigação disponível é ainda muito incipiente. Embora não tenhamos base empírica para o afirmar categoricamente acreditamos que hoje são já muitos os profissionais que usam a Internet no seu trabalho com clientes. Igualmente serão numerosos os clientes que autonomamente recorrem à Internet para obterem informações relevantes sobre os seus problemas de vida. Uma vantagem da Internet, relativamente a outras modalidades de ajuda psicológica, é a sua grande acessibilidade, praticamente independente dos constrangimentos do tempo e do espaço. Num momento, como aquele que atravessamos atualmente no nosso país, em que muitos precisam de apoio para lidarem com as circunstâncias de vida adversa com que se debatem (v.g., desemprego, reemprego) a Internet pode ser um complemento útil para colmatar a falta de recursos humanos disponíveis.

Outra razão, para a falta de investigação nesta área, tem a ver com a inexistência de instrumentos de investigação adequados. Neste estudo apresentámos os primeiros resultados respeitantes à adaptação de duas escalas que avaliam as atitudes dos clientes a respeito das modalidades de aconselhamento online e tradicional. Os resultados que obtivemos são encorajadores tendo-se conseguido replicar a estrutura fatorial do instrumento original na amostra Portuguesa. Dois componentes Valor e Desconforto foram solidamente estabelecidos e ambos demonstraram bons níveis de precisão psicométrica. Não foram encontradas diferenças sexuais nas subescalas e as relações dos scores com a idade revelaram-se marginais. Todavia, nesta amostra de respondentes desempregados, há alguma evidência de que se atribui um maior valor ao aconselhamento face-a-face do que ao aconselhamento online. Esta tendência não foi replicada para a dimensão de desconforto com o aconselhamento.

As atitudes a respeito do aconselhamento online pesarão na decisão que os clientes tomarão se lhes for facultado o acesso a esta modalidade de ajuda. Avaliar as atitudes dos clientes a respeito do aconselhamento online poderá prevenir a sua insatisfação e o abandono prematuro do processo de ajuda. Estudar quais as estratégias que podem ser usadas para modificar as crenças não adaptativas e as opiniões incorretas dos clientes a respeito do aconselhamento online é uma área que urge examinar em futuras investigações.

Embora seja importante replicar os resultados que obtivemos neste estudo em outras amostras e com outras tipologias de indivíduos, conclui-se que as escalas analisadas permitem obter medidas válidas das atitudes acerca do aconselhamento e que delas podemos extrair implicações clínicas práticas relevantes

\section{Referências}

Ajzen, (2001). Nature and operation of attitudes. Annual Review of Psychology, 52, 27-58. http://dx.doi.org/10.1146/annurev.psych.52.1.27

APS (2012). Internet supported psychological interventions. Extraído de www.psychology.org.au

Andersson, G., \& Cuijpers, P. (2009). Internet-Based and Other Computerized Psychological Treatments for Adult Depression: A Meta-Analysis. Cognitive Behaviour Therapy, 38, 196-205.

http://dx.doi.org/10.1080/16506070903318960

Anthony, K. (2003). The use and role of technology in counselling and psychotherapy. In S. Goss \& Anthony (Eds.), Technology in counselling and psychotherapy (pp. 13-35). New York: Palgravre Macmillan.

Anthony, K. \& Goss, S. (2003). Introduction. In S. Goss \& Anthony (Eds.), Technology in counselling and psychotherapy (pp. 1-12). New York: Palgravre Macmillan.

Barak, A. (1999). Psychological applications on the Internet: A discipline on the threshold of a new millennium. Applied \& Preventive Psychology, 8, 231-

245. http://dx.doi.org/10.1016/S0962-1849(05)800381

Barak, A., Hen, L., Boniel-Nissim, M., \& Shapira, N. (2008). A comprehensive review and a meta-analysis of the effectiveness of Internet-based psychotherapeutic interventions. Journal of Technology in Human Services, 26, 109-160. http://dx.doi:10.1080/15228830802094429

Barak, A., Klein, B., \& Proudfoot, J. (2009). Defining internet-supported therapeutic interventions. Annals of Behavioral Medicine, 38, 4-17. http://dx.doi:10.1007/s12160-009-9130-7

Rochlen, A., Beretvas, S., \& Zack, J. (2004). The on-line and face-to-face counseling attitude scales: A validation study. Measurement and Evaluation in Counseling and Development, 37, 95-111.

Rochlen, A., Zack, J., \& Speyer, C. (2004). Online therapy: Review of relevant definitions, debates, and current empirical support. Journal of Clinical Psychology, 60, 269-283. http://dx.doi.org/10.1002/jclp.10263

Tate, D. F., Finkelstein, E. A., Khavjou, O., \& Gustafson, A. (2009). Cost effectiveness of Internet interventions: Review and recommendations. Annals of Behavioral Medicine, 38, 40-45.

http://dx.doi.org/10.1007/s12160-009-9131-6

Taveira, M., C. \& Silva, J. T. (2011). O uso de tecnologia na intervenção vocacional: implicações para a teoria e prática. In M. C. Taveira \& J. T. Silva (Eds.). Psicologia vocacional. Perspectivas para a intervenção (pp. 93-138). Coimbra: Imprensa da Universidade de Coimbra.

\section{Agradecimentos}

Os autores agradecem a colaboração da/os psicóloga/os na administração dos questionários e a todos os indivíduos que voluntariamente acederam participar nesta investigação. 